\title{
Producir textos en los inicios de la alfabetización: el impacto de diferentes enfoques pedagógicos en la expresión evaluativa ${ }^{1}$
}

\author{
Gabriela Zuccalá \\ Universidad Nacional de La Plata
}

Ana Sandbank

Beit Berl Academic College y Levinsky College of Education

(Texto recibido el 4 de diciembre de 2017; aceptado el 7 de mayo de 2018)

DOI: http://doi.org/10.5565/rev/jt13.771

Resumen: Esta investigación se propuso analizar las expresiones evaluativas de textos narrativos, producidos por niños del nivel inicial y primer grado a partir de una historieta, en tres modalidades: oral, dictado a un adulto y escrito. Participaron 33 niños del nivel inicial y 32 de primer grado educados con enfoque constructivista y 30 niños del nivel inicial y 28 de primer grado educados con enfoque formal. Los resultados muestran un porcentaje significativamente mayor de expresiones evaluativas en los textos producidos por niños educados con enfoque constructivista en ambos grupos de edad y en todas las modalidades. El análisis cualitativo se centró en la variedad de expresiones evaluativas. Las implicaciones educativas de estos resultados son discutidas.

Palabras clave: expresión evaluativa, enfoque pedagógico, alfabetización, producción de textos narrativos

Summary: The aims of this study are to analyze the impact of the approach for teaching literacy on the evaluative expressions in narrative texts, based on a comic strip, and to compare the texts produced by preschoolers and first graders in three modalities: oral, dictated to an adult and written. Participants were 33 preschoolers and 32 first graders attending schools with a 'constructivist' approach to teaching literacy, as well as 30 preschool children and 28 first graders attending schools with a formal systematic approach. Results show a significantly higher percentage of evaluative expressions in the texts produced by children educated with a constructivist approach in both age groups in the three modalities. A qualitative analysis shows a variety of evaluative expressions. The educational implications of these findings are discussed.

Keywords: evaluative expressions, pedagogic approach, literacy, narrative texts production

Résumé : L'étude a pour objectif d'analyser 1 impact de deux approches pédagogiques pour l'enseignement de la langue écrite: constructiviste et formelle/systématique. Il s'agit de comparer des textes narratifs produits sur une base de bande dessinée, par des enfants d'âge préscolaire avec ceux produits par des enfants de première année. Et ce en trois modalités; orale, dictée à un adulte et écrite. L'approche constructiviste a été testée sur un groupe composé de 33 enfants d'un niveau initial (préscolaire) et 32 élèves de première année d'école élémentaire. La pédagogie formelle/ systématique quant-à-elle est expérimentée sur un ensemble de 30 enfants de niveau initial (pré- 
scolaire) et 28 élèves de première année d'école élémentaire. Les résultats de cette étude démontrent que l'apprentissage par la pédagogie constructiviste développe un niveau d'expression évaluative significativement plus élevé dans les textes produits, quel que soit le groupe d'âge. L'analyse qualitative dépeint une grande variété d'expressions évaluatives. Les implications éducatives de ces résultats sont développées dans l'article.

Mots-clés : expressions évaluatives, approche pédagogique, alphabétisation, production de textes narratifs

\section{Introducción}

La investigación psicogenética acerca de la lengua escrita se concentró en las ideas de los niños acerca de la escritura como sistema de notación (Ferreiro y Teberosky, 1979; Pontecorvo y Rossi, 2012; Tolchinsky, 2003). Paralelamente, el concepto de alfabetización o 'literacidad' fue ampliándose, involucrando además la investigación acerca del lenguaje que se escribe (Blanche-Benveniste, 1998; Pontecorvo y Orsolini, 1996), prácticas discursivas culturales (Heath, 1983), entre pares (Cekaite, Blum-Kulka, Grøver, y Teubal, 2014) y con adultos (Wells, 2009), modos de 'lectura' de libros previos a la lectura convencional (Sulzby, 1994), y la actividad metalingüística (Astington, 2000; Brockmeier y Olson, 2002; Castedo, 2001; Homer, 2009). Ello permitió además integrar el desarrollo del lenguaje oral con la alfabetización (Ravid y Tolchinsky, 2002; Snow, 2017). Uno de los dominios comunes al lenguaje y la alfabetización es la capacidad narrativa.

La evolución de la capacidad narrativa fue ampliamente estudiada en producciones orales, considerando diferentes aspectos: organización del texto, relaciones entre las formas lingüísticas y las funciones que ellas cumplen en la narración (Berman y Slobin, 1994), el contenido (Bruner, 2002; Nelson, 2014), la coherencia (Nicolopoulou, 2008).

Algunas investigaciones sobre textos narrativos escritos se focalizaron en las convenciones del sistema de escritura (Ferreiro, Pontecorvo, Ribeiro Moreira, y García Hidalgo, 1996; Sandbank, 2001). Otras, en las características del discurso narrativo (Rossi, Pontecorvo, López-Orós, y Teberosky, 2000), así como en la contribución de propuestas didácticas a la calidad de los textos (Sepúlveda y Teberosky, 2011).

Este artículo presenta una investigación acerca de los textos producidos por niños del último año de la educación inicial y primer grado en tres modalidades: oral, dictado del texto a un adulto, y escritura. Las tres modalidades fueron presentadas como una secuencia fija, considerada como un andamiaje para la escritura de textos (Myhill y Jones, 2009). El análisis de los textos se concentra en las expresiones evaluativas. 
El concepto de evaluación fue introducido por el sociolingüista Labov al investigar narrativas orales de adolescentes de clase baja (Labov y Waletsky, 1967). Su análisis se basó en una distinción entre cláusulas referenciales que constituyen el 'esqueleto' de la narración y cláusulas evaluativas que revelan la interpretación del narrador y el modo de dirigir la atención de la audiencia hacia ciertos aspectos del relato. Investigaciones posteriores han modificado la dicotomía referencial-evaluativo, caracterizando las expresiones evaluativas como un continuo que va de las acciones a aspectos interpretativos (Kupersmitt y Sandbank, 2010; Shiro, 2003; Veneziano, 2016). Por ejemplo, una acción 'ir (al trabajo)’ puede adquirir un valor evaluativo agregado en ciertos contextos 'irse (de su casa)', debido a que no solo expresa una acción sino también aspectos afectivos (Astington, 2000).

Estudios evolutivos han mostrado que la evaluación aumenta con la edad. Niños de 4 a 7 años pueden atribuir estados mentales a los protagonistas de una historia (Richner y Nicolopoulou, 2001), pero es alrededor de los 8-9 años que usan esos estados internos para explicar las conductas de los protagonistas (Bamberg y Damrad-Frye, 1991; Berman y Slobin, 1994). Es aún más tarde que pueden expresarse las diferentes perspectivas de los protagonistas sobre un mismo evento (Betancourt y Montes, 2013; Kuntay y Nakamura, 2004).

La inclusión de formas con función evaluativa en las narraciones parece también depender del contexto en el que se producen los textos. Por ejemplo, se tiende a incluir expresiones evaluativas cuando se conversa acerca de las causas de las acciones de los protagonistas (Veneziano, 2016). La inclusión de expresiones evaluativas parece además estar relacionada con prácticas educativas como cuando la narración se basa en un cuento leído (de la Vega y Alvarado, 2013; Hess, 2013). El objetivo de esta investigación fue analizar el impacto de diferentes enfoques educativos en la expresión de la evaluación en narraciones producidas en el último año de la escuela inicial y el primer grado de la escuela elemental ${ }^{2}$ en las tres modalidades.

\section{La caracterización de los enfoques educativos}

La caracterización de los enfoques educativos se realizó a partir de entrevistas y observaciones de las prácticas usuales relacionadas con la alfabetización. Se definieron dos enfoques diferentes: constructivista y formal ${ }^{3}$.

En las clases de enfoque constructivista se propiciaba el contacto con textos de circulación social: carteles y producciones con escrituras convencionales y no 
convencionales, de adultos y de niños por igual, con escrituras de palabras, de letras y de textos completos, así como la revisión con participación activa y reflexiva de los alumnos. Los niños participaban asiduamente en situaciones de lectura: escuchaban leer a un adulto o leían por sí mismos con diversos propósitos: buscar información específica, saber más sobre un tema, disfrutar de una obra literaria, etc. La biblioteca del aula contenía textos de géneros diversos y se utilizaba cotidianamente.

En las clases con enfoque formal se ejercitaban el conocimiento de letras y la conciencia fonológica, las correspondencias fonográficas y las reglas de combinación, en forma sistemática y gradual. Los niños realizaban tareas de copia o escritura de listas de palabras y oraciones. La escritura de textos se posponía hasta haber logrado la escritura convencional. Los textos presentes en el aula estaban escritos de manera convencional (Grunfeld y Molinari, 2017). La enseñanza de la lectura se centraba en el descifrado del texto: leer sílabas o palabras. Las bibliotecas incluían textos de literatura infantil que solo leía la educadora.

Investigaciones realizadas en otros países han develado diferencias similares, aunque no idénticas, entre los enfoques pedagógicos adoptados para la alfabetización inicial. (Tolchinsky, Bigas, y Barragán, 2012).

\section{Metodología}

Participantes. Participaron 33 niños del nivel inicial y 32 de primer grado educados con enfoque constructivista y 30 niños del nivel inicial y 28 de primer grado educados con un enfoque formal. La condición socio-económica y cultural de los participantes se definió como desfavorecida, considerando el nivel de escolaridad y la ocupación de los padres. Los criterios de participación fueron: a) un mínimo de $70 \%$ de asistencia a clase, y b) no presentar dificultades de aprendizaje. Las siguientes tablas presentan la distribución de los participantes. 
Tabla 1. Promedio de edad de los niños entrevistados (rango entre paréntesis) y desviación estándar (SD) por nivel escolar, enfoque pedagógico y género

\begin{tabular}{|c|c|c|c|c|c|c|c|c|}
\hline \multirow{2}{*}{$\begin{array}{l}\text { Nivel } \\
\text { Enfoque }\end{array}$} & \multicolumn{4}{|l|}{ Inicial } & \multicolumn{4}{|c|}{ Primer grado } \\
\hline & $\begin{array}{l}\text { Construct } \\
(\mathrm{N}=33)\end{array}$ & vista & $\begin{array}{l}\text { Formal } \\
(\mathrm{N}=30)\end{array}$ & & $\begin{array}{l}\text { Construc } \\
(\mathrm{N}=32)\end{array}$ & vista & $\begin{array}{l}\text { Formal } \\
(\mathrm{N}=28)\end{array}$ & \\
\hline Género & $\begin{array}{l}\text { Niñas } \\
(\mathrm{N}=17)\end{array}$ & $\begin{array}{l}\text { Niños } \\
(\mathrm{N}=17)\end{array}$ & $\begin{array}{l}\text { Niñas } \\
(\mathrm{N}=12)\end{array}$ & $\begin{array}{l}\text { Niños } \\
(\mathrm{N}=18)\end{array}$ & $\begin{array}{l}\text { Niñas } \\
(N=14)\end{array}$ & $\begin{array}{l}\text { Niños } \\
(\mathrm{N}=18)\end{array}$ & $\begin{array}{l}\text { Niñas } \\
(\mathrm{N}=19)\end{array}$ & $\begin{array}{l}\text { Niños } \\
(\mathrm{N}=9)\end{array}$ \\
\hline Promedio & $5 ; 8$ & $5 ; 9$ & $6 ; 1$ & $5 ; 9$ & 7 & $6 ; 7$ & $7 ; 1$ & $6 ; 7$ \\
\hline Rango & $(5 ; 6-6 ; 2)$ & $(5 ; 4-6 ; 4)$ & $(5 ; 5-6 ; 3)$ & $(5 ; 4-5 ; 6)$ & $(6 ; 5-7 ; 3)$ & $(6 ; 4-7 ; 3)$ & $(6 ; 2-7 ; 9)$ & $(6 ; 4-7 ; 3)$ \\
\hline SD & 3.65 & 4.5 & 3.51 & 4.19 & 3.02 & 4.87 & 3.54 & 3.08 \\
\hline
\end{tabular}

Procedimiento. La tarea propuesta se iniciaba mostrando la siguiente secuencia de viñetas extraídas de la historieta 'Mafalda' (Quino, 2011; p. 388).

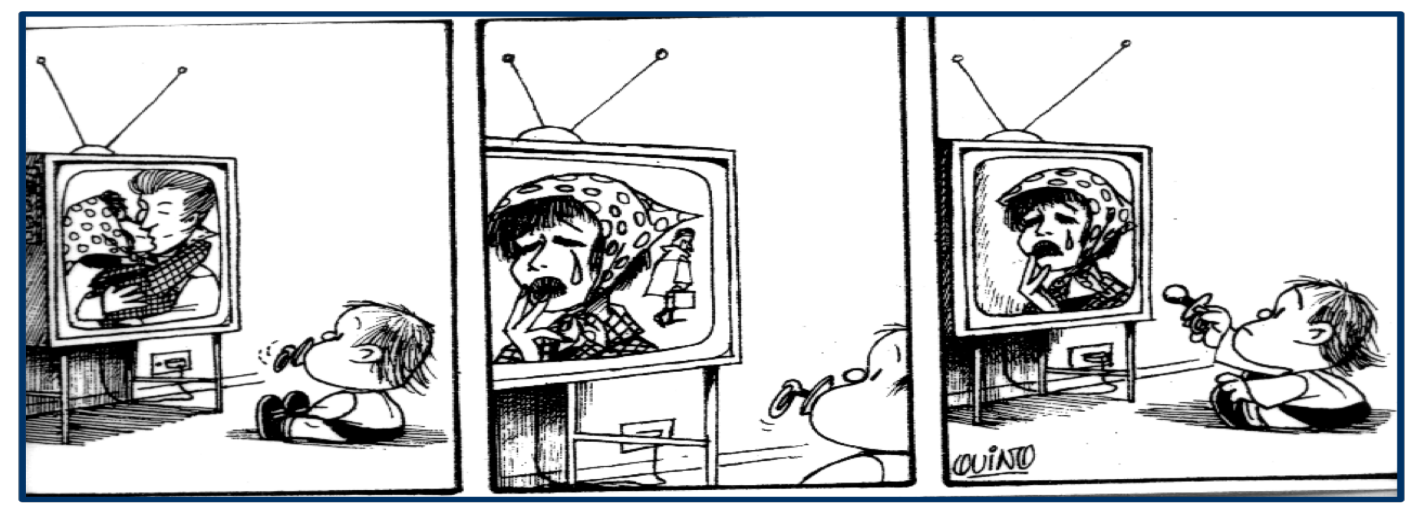

Imagen1. 'Mafalda' (Quino, 2011, p. 338)

La historieta presentada a los niños se organiza en dos planos de acción paralelos, marcados por un contraste en el escenario y en los personajes (Hoey, 2001). Uno de ellos ocurre en la televisión, en el que participan un hombre y una mujer. El otro es el espacio donde está el bebé. Ambos planos se integran en el final con el intento del bebé de darle su chupete a la mujer que llora.

Se presentaron tres consignas a cada niño en el mismo orden ${ }^{4}$.

Texto oral: "Te voy a mostrar una historieta. Contame lo que ocurre en ella".

Texto dictado al entrevistador: "Ya me contaste lo que ocurre en la historieta. Ahora dictame como lo escribirías para que tus compañeros entiendan la historieta sin verla. Vos me dictás y yo escribo". Al finalizar la escritura, el entrevistador leía el texto en 
voz alta y le preguntaba al niño si estaba correcto, efectuando agregados o cambios si el niño lo solicitaba.

Texto escrito: "Ahora escribí vos lo que ocurre en la historieta" (en imprenta o manuscrita).

El Corpus. Los textos orales fueron grabados y posteriormente transcriptos textualmente. Los textos dictados fueron escritos por el entrevistador que adoptó el papel de 'escriba'. Los textos escritos fueron transcriptos en una versión normalizada. Para ello se les pedía a los niños que leyeran el texto señalando lo que iban leyendo mientras el entrevistador lo escribía. El siguiente es un ejemplo de transcripción de un segmento del texto original del niño (en mayúscula) y debajo las 'lectura' del sujeto (en minúscula) al interpretar lo escrito:

M Q W O M

El bebé está mirando

N I A U n W

se estaban dando un beso

B N Q e N

estaba llorando [...].

[Bru, m, I, Constructivista, escrito.$^{5}$

Este ejemplo de escritura se caracteriza por representar diferentes enunciados con diferentes combinaciones de letras, sin correspondencia fonográfica. El siguiente ejemplo muestra una escritura más avanzada con una correspondencia silábica: $U=u n, B=b e B=b e, T=t e, E=l e$, $\mathrm{I}=\mathrm{vi}, \mathrm{S}=\mathrm{si}, \mathrm{O}=$ ón, aunque no todas las palabras que la niña 'lee' están escritas ${ }^{6}$.

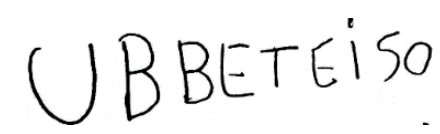

Imagen 2. Un bebé está mirando la televisión. [Wan, f, P, Formal, escrito]

La tabla 2 resume el corpus de textos analizados. 
Tabla 2. Distribución del número de textos en cada modalidad por nivel escolar y enfoque pedagógico

\begin{tabular}{|c|c|c|c|c|c|}
\hline Nivel & Inicial & & Primer grado & & Total \\
\hline Enfoque & $\begin{array}{l}\text { Constructivista } \\
\mathrm{N}=33\end{array}$ & $\begin{array}{l}\text { Formal } \\
\mathrm{N}=29\end{array}$ & $\begin{array}{l}\text { Constructivista } \\
\mathrm{N}=32\end{array}$ & $\begin{array}{l}\text { Formal } \\
\mathrm{N}=28\end{array}$ & $\mathrm{~N}=122$ \\
\hline \multicolumn{6}{|c|}{ Modalidad } \\
\hline Oral & 33 & 29 & 32 & 28 & 122 \\
\hline Dictado & 26 & 25 & 27 & 21 & 99 \\
\hline Escrito & 32 & 23 & 32 & 26 & 113 \\
\hline Total & 91 & 77 & 75 & 91 & 334 \\
\hline
\end{tabular}

De los datos presentados se desprende que todos los participantes produjeron la narrativa oral en ambos enfoques. La producción de textos dictados y escritos fue más frecuente en enfoque constructivista, siendo más los escritos que los dictados en ambos niveles. También en primer grado con enfoque formal se produjeron más textos escritos que dictados, mientras que en el nivel inicial fueron más los dictados que los escritos.

\section{Resultados: EI análisis de las expresiones evaluativas}

Las siguientes categorías de expresiones evaluativas fueron elaboradas a partir del análisis de todos los textos producidos en las tres modalidades. Cada categoría se presenta relacionando las formas lingüísticas con su función evaluativa.

1. Actividades con valor evaluativo agregado. Verbos que expresan sentimientos. Por ejemplo:

La mamá está llorando y el bebé le está dando su chupete. [Bra, m, P, Constructivista, dictado].

2. Estados afectivos. Estados que expresan sentimientos en forma explícita cuando los niños hacen alusión a estar triste o estar feliz, así como a través de la descripción de una situación objetiva como 'estar sola'. Por ejemplo:

La señora está triste porque se va el señor y después está sola y llorando. Y cuando vuelve el señor está feliz [Mai, f, I, Constructivista, oral]

3. Intensificadores. Repeticiones o términos que expresan intensidad. Por ejemplo:

Extrañó a su esposo tanto tanto [Ale,f, I, Constructivista, oral].

4. Estados mentales. Verbos mentales como pensar y creer, reflejan la interpretación que los niños realizan de las acciones de los personajes. Por ejemplo: 
La mamá está llorando y el bebé le está dando su chupete. Piensa que el chupete le va a hacer reír a la mujer. [Bra, m, P. Constructivista, dictado]. En el siguiente ejemplo (Imagen 3) de un texto escrito se expresan estados mentales: creer y pensar.

$$
\begin{aligned}
& \text { GILLE ESTA VIENDO UNA NOVELA } \\
& \text { Y LA CHICA QUE ESTA ENLA NOUELA } \\
& \text { SE CREE QUE ES SU MA MA } \\
& \text { Y GILLE SE SACA EL CAUPETE Y } \\
& \text { SE NALA A LA CHICA MEE ES TA ES LP } \\
& \text { TELE LO MI'BA SERIOO PENSAN DO } \\
& \text { QUE ES SU MAMA }
\end{aligned}
$$

Imagen 3. Guille está viendo una novela y la chica que está en la novela se cree que es su mamá y Guille se saca el chupete y señala a la chica que está en la tele. Lo mira serio pensando que es su mamá [Yam, f. P, Constructivista, escrito].

En el siguiente ejemplo el mismo verbo 'ver' se interpreta en dos modos diferentes.

Acá ve una película. Después ve que la mujer de la tele está llorando. Quiere darle el chupete para que no llore más. [Sof, f, I, Constructivista, oral].

El primer 've' se refiere a una percepción, mientras que el segundo 've' significa además darse cuenta, que indica un estado mental, es decir que cumple una función evaluativa.

5. Intenciones y propósitos. La función evaluativa se expresa además a través de perífrasis modales, “construcciones en que en las que la acción del infinitivo es vista por el hablante como obligatoria, necesaria, deseada, posible, probable, etc." (Bosque y Demonte, 1999; p. 3347), como se ilustra en los siguientes ejemplos.

El bebé está viendo besarse con el chico y se tuvo que despedir el chico [Lar, f. I, constructivista, oral].

y el bebé quiere darle un beso a la chica para que se ponga feliz. [Lar, f. I, Constructivista, oral]

Acá ve una película. Después ve que la mujer de la tele está llorando. Quiere darle el chupete para que no llore más. [Sof, f, I, Constructivista, oral].

Entre los textos analizados algunos incluyen múltiples expresiones evaluativas, como puede verse en los siguientes ejemplos del grupo de educación inicial. El primero es un relato oral y el segundo un texto escrito.

Esta historia empieza así: un bebé estaba un día sentado mirando una novela. El chico se tuvo que despedir y la chica lloró. Entonces el bebé se sacó el chupete 
porque pensaba que la chica de verdad. Entonces se sacó el chupete para dárselo, para que no siga llorando [Lar, f, I, Constructivista, oral].

$$
\begin{aligned}
& \text { EL OMBRE LA DEJO } \\
& \text { Y LA MUGER SE QUEDO YORANBO } \\
& \text { PORQUE EL OMBRE SE FUE A TRAAAJAR } \\
& \text { Y GILL LE D:O EL CHUPETE } \\
& \text { PORQUE LE DIO TRISTESA }
\end{aligned}
$$

Imagen 4. El hombre la dejó y la mujer se quedó llorando, porque el hombre se fue a trabajar y Guille le dio el chupete, porque le dio tristeza [Bel, f, I, Constructivista, escrito].

El texto oral presenta una expresión modal que implica obligación 'se tuvo que despedir' que puede considerarse como la causa de la actividad que expresa un estado afectivo 'Iloró'. A continuación, se presenta una causa, que incluye un estado mental 'porque pensaba que la chica de verdad'. Finalmente se presentan dos cláusulas subordinadas que expresan propósitos o intenciones 'para dárselo, para que no siga llorando'.

El segundo ejemplo presenta una acción 'la $\mathbf{d e j o ́}$ ' con un valor evaluativo agregado (negativo), una actividad 'quedarse llorando' que expresa un estado afectivo. Se presentan además dos causas. La primera es un evento 'el hombre se fue a trabajar' mientras que la segunda se refiere a un estado mental-afectivo 'le dio tristeza'. Los textos presentados muestran no solo variedad, sino también densidad evaluativa (Kupersmitt y Sandbank, 2010). Otros textos, en cambio, incluyeron una única referencia evaluativa, como el siguiente:

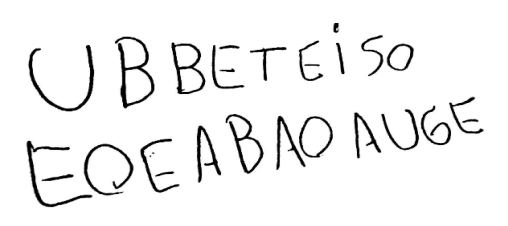

Imagen 5. Un bebé está mirando la televisión. La mujer está llorando y se puso la mano en la cara [Wan, f, P, Formal, escrito]

Algunos relatos incluyeron solo acciones, sin ninguna expresión evaluativa, como puede verse en los siguientes ejemplos de dos niñas de primer grado.

El bebé mira la tele. El bebé se quita el chupete. Prendió la tele con el control remoto.

[Ann, f, P, Formal, oral].

El bebé está viendo televisión. Una parte donde un hombre se va. [Mar, f, P. Constructivista, oral]. 
Las tablas 3 y 4 presentan la distribución de textos de acuerdo a la inclusión de algún contenido evaluativo según modalidad, enfoque pedagógico y nivel escolar (inicial y primaria respectivamente).

Tabla 3. Distribución del porcentaje de textos de acuerdo con la inclusión de contenido evaluativo por enfoque pedagógico (constructivista y formal) y modalidad (oral, dictado, escrito) en nivel inicial

\begin{tabular}{lllllllll}
\hline Enfoque & \multicolumn{1}{l}{$\begin{array}{l}\text { Constructivista } \\
\mathrm{N}=33\end{array}$} & \multicolumn{7}{c}{$\begin{array}{l}\text { Formal } \\
\mathrm{N}=30\end{array}$} \\
\hline $\begin{array}{l}\text { Modalidad } \\
\text { Evaluación }\end{array}$ & Oral & Dictado & Escrito & Total & Oral & Dictado & Escrito & Total \\
\hline Incluye & 48 & 24 & 42 & 38 & 24 & 3 & 6 & 11 \\
No Incluye & 52 & 55 & 55 & 54 & 73 & 81 & 70 & 75 \\
No Producidos & 0 & 21 & 3 & 8 & 3 & 16 & 24 & 14 \\
Total & 100 & 100 & 100 & 100 & 100 & 100 & 100 & 100 \\
\hline
\end{tabular}

Tabla 4. Distribución del porcentaje de textos (de acuerdo con la inclusión de contenido evaluativo por enfoque pedagógico (constructivista y formal) y modalidad (oral, dictado, escrito) en primer grado.

\begin{tabular}{lllllllll}
\hline Enfoque & \multicolumn{2}{l}{ Constructivista } & \multicolumn{7}{c}{$\begin{array}{l}\text { Formal } \\
\mathrm{N}=32\end{array}$} & & \multicolumn{7}{c}{ (28 } \\
\hline $\begin{array}{l}\text { Modalidad } \\
\text { Evaluación }\end{array}$ & Oral & Dictado & Escrito & Total & Oral & Dictado & Escrito & Total \\
\hline Incluye & 53 & 47 & 53 & 51 & 11 & 11 & 29 & 16.5 \\
No Incluye & 47 & 37 & 47 & 44 & 89 & 64 & 64 & 72.5 \\
No producidos & 0 & 16 & 0 & 5 & 0 & 25 & 7 & 11 \\
Total & 100 & 100 & 100 & 100 & 100 & 100 & 100 & 100 \\
\hline
\end{tabular}

Los datos muestran que la inclusión de contenido evaluativo aumenta con la edad y es mayor en el enfoque constructivista que en el formal. Los niños más pequeños de las escuelas con enfoque constructivista produjeron más textos con contenido evaluativo que los de primer grado de las escuelas con enfoque formal.

Para evaluar si existen diferencias significativas en la proporción de expresiones evaluativas de los textos producidos se calculó la proporción de cláusulas con contenido 
evaluativo del total de cláusulas ${ }^{7}$. El análisis de varianza ANOVA entre 2 (edad) X 2 (enfoque) X 3 (modalidad como medidas repetidas) muestra una diferencia significativa por enfoques pedagógicos $(\mathrm{F}(1,82)=4.53, \mathrm{p}<.05)$. Se encontraron también diferencias por modalidad $(\mathrm{F}(2,164)=3.68, \mathrm{p}<.05)$. En el nivel inicial, la proporción de cláusulas con contenido evaluativo es ligeramente mayor en los textos dictados que en los escritos y mayor en los escritos que en los orales, mientras que en el grupo inicial con educación formal su proporción es mayor en los escritos. En cambio, en primer grado se observó una proporción mayor de cláusulas con contenido evaluativo en los textos escritos en ambos enfoques. No se encontraron interacciones significativas entre enfoque, nivel escolar y modalidad. Es importante recordar que el porcentaje de textos escritos tanto en el nivel inicial como en primer grado fue menor en el enfoque formal que en el enfoque constructivista (ver tabla 2).

Además del contenido evaluativo expresado en las cláusulas, la organización misma del texto puede cumplir también una función evaluativa. Por ejemplo, se expresa un modo de referirse a los acontecimientos relatados cuando se establecen comparaciones o contrastes entre diferentes segmentos textuales (Reinhart, 1985). En el caso de la historieta, se trata del diseño de un paralelismo entre las acciones de los protagonistas que aparecen en la pantalla de televisión -es decir uno de los planos en los que ocurre la acción - y las acciones del bebé, otro plano. Esta distinción se tomó como base para el análisis de la organización que expresa la interpretación de la historieta en los textos producidos.

Textos que integran ambos planos de acción. Algunos textos incluyeron ambos planos de acción (bebé, novela) explicitando la vinculación de los personajes y sucesos de la novela con el bebé y su intención de 'participar' en la ficción televisiva, dándole el chupete a la mujer, así como el punto de vista del bebé acerca de lo que les ocurre a los personajes. Por ejemplo: 


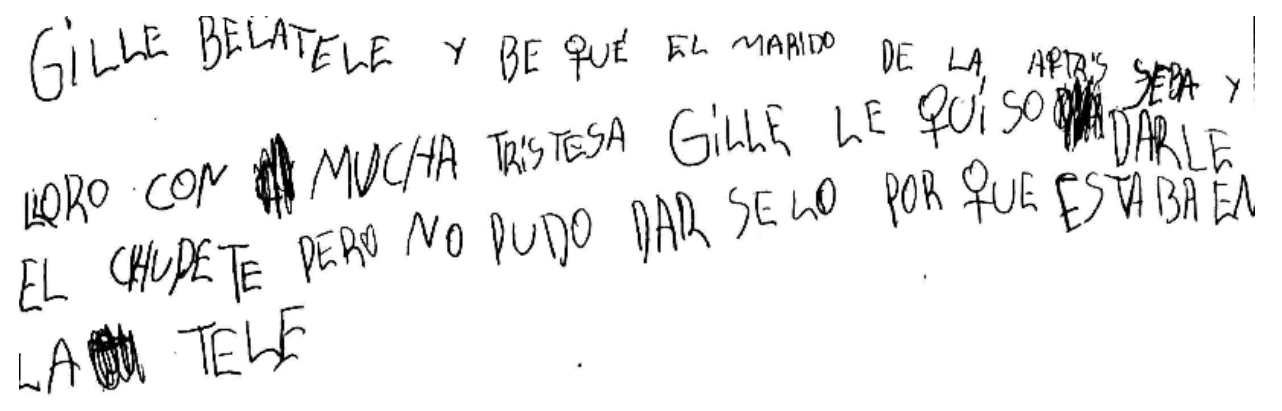

Imagen 6. Guille ve la tele y ve que el marido de la actriz se va y lloró con mucha tristeza. Guille quiso darle el chupete. Pero no pudo dárselo porque estaba en la tele [Car, f, P, Constructivista, escrito]

Textos que toman los dos planos de manera yuxtapuesta. Otros textos incluyeron ambos planos, pero sin referencias a aquellas acciones que los conectan. Por ejemplo:

Un bebe está mirando el televisor Y una mujer y un hombre se están besando

Y la mujer se puso los dedos en la cara y estaba llorando. Y él bebe puso el chupete al lado del televisor [Fer, v, P, Formal, oral]

El texto presentado incluye a todos los protagonistas y sus actividades, pero no hay referencia al motivo por el cual se saca el chupete, ni a su intención o el punto de vista del bebé sobre lo que ve que sucede. En el siguiente texto escrito se ven los dos planos que aparecen en la pantalla y a las del bebé, pero no hace referencia al punto de vista del bebé sobre los hechos.

$$
\begin{aligned}
& \text { LLORANDO } \\
& \text { SENOA } \\
& \text { CAMINANO } \\
& \text { BEBE } \\
& \text { MIRANDO } \\
& \text { LAIELE }
\end{aligned}
$$

Imagen 7. Llorando. Señor caminando. Bebé mirando la tele. [Ner, f, P, Formal, escrito]

Textos organizados en un plano. En algunos textos se reconstruyeron solo los sucesos de uno de los planos, sin ninguna mención al otro. Por ejemplo:

Primero un señor está con una chica, después el señor se fue. Y después la chica está llorando [Mar, m, I, Constructivista, oral].

El ejemplo presentado es un texto narrativo oral centrado en los personajes que aparecen en la televisión y sus acciones, exclusivamente. En el siguiente ejemplo se toma el otro plano: 
El bebé está mirando la tele y está chupando el chupete [Jos, v, P, Constructivista, dictado]

Las siguientes tablas (tablas 5 y 6) presentan la distribución de los textos en relación a los planos de la historieta consideradas, de acuerdo con la edad y el enfoque pedagógico.

Tabla 5. Porcentaje de textos por planos mencionados para cada enfoque (constructivista o formal) y modalidad (oral, dictado, escrita) en nivel inicial.

\begin{tabular}{lllllllll}
\hline Enfoque & \multicolumn{2}{l}{$\begin{array}{l}\text { Constructivista } \\
\mathrm{N}=33\end{array}$} & & \multicolumn{5}{c}{$\begin{array}{c}\text { Formal } \\
\mathrm{N}=29\end{array}$} \\
$\begin{array}{lllllll}\text { Modalidad } \\
\text { Planos }\end{array}$ & Oral & Dictado & Escrito & Total & Oral & Dictado & Escrito & Total \\
\hline Integrados & 21 & 15 & 24 & 20 & 17 & 0 & 3 & 7 \\
Yuxtapuestos & 49 & 15 & 49 & 37.5 & 40 & 0 & 30 & 23 \\
Un Plano & 30 & 48 & 24 & 34.5 & 40 & 84 & 43.5 & 55.5 \\
No Producidos & 0 & 21 & 3 & 8 & 3.5 & 16 & 23.5 & 14.5 \\
Total & 100 & 100 & 100 & 100 & 100 & 100 & 100 & 100 \\
\end{tabular}

Tabla 6. Porcentaje de textos por planos mencionados, para cada enfoque (constructivista o formal) y modalidad (oral, dictado, escrita) en primer grado.

\begin{tabular}{lllllllll}
\hline Enfoque & \multicolumn{2}{l}{$\begin{array}{l}\text { Constructivista } \\
\text { N=32 }\end{array}$} & \multicolumn{7}{c}{$\begin{array}{l}\text { Formal } \\
\text { N=28 }\end{array}$} \\
\hline $\begin{array}{l}\text { Modalidad } \\
\text { Planos }\end{array}$ & Oral & Dictado & Escrito & Total & Oral & Dictado & Escrito & Total \\
\hline Integrados & 44 & 44 & 53 & 47 & 11 & 14.5 & 14.5 & 13 \\
Yuxtapuestos & 47 & 21 & 19 & 29 & 43 & 14.5 & 35.5 & 31 \\
Un Plano & 9 & 19 & 28 & 19 & 46 & 46 & 43 & 45 \\
$\begin{array}{l}\text { No } \\
\text { Producidos }\end{array}$ & 0 & 16 & 0 & 5 & 0 & 25 & 7 & 11 \\
Total & 100 & 100 & 100 & 100 & 100 & 100 & 100 & 100 \\
\hline
\end{tabular}

De los datos presentados surge que las posibilidades de los niños de construir textos que consideren los dos planos, integrándolos en el relato, aumenta en primer grado, tanto en escuelas constructivistas como en escuelas formales. En ambos niveles escolares la mención 
de los dos planos integrados es mayor en los textos de los niños educados con un enfoque constructivista. Más aún, los niños de la educación inicial con enfoque constructivista presentaron una proporción mayor de textos con planos integrados que los niños de primeros grados formales.

La organización de la narración en planos integrados fue más frecuente en los textos escritos en ambos niveles educativos caracterizados por un enfoque constructivista. En cambio, en el enfoque formal la integración de los planos fue más frecuente en los textos orales en el nivel inicial, mientras que en primer grado fue idéntica en los orales y los escritos.

\section{Conclusiones}

El análisis presentado se concentró en dos aspectos de la interpretación de la historieta: el contenido evaluativo y los planos en los que se desarrolla la historia, desde una perspectiva de análisis cuantitativa y cualitativa.

El análisis cuantitativo reveló que los niños más pequeños de clases con enfoque constructivista lograron un mejor desempeño en la inclusión de expresiones evaluativas y la integración de los planos de la acción, que los niños que participan de un enfoque pedagógico formal. Desde un punto de vista cualitativo, se encontraron formas variadas que los niños utilizaron para expresar contenidos evaluativos también diversos: desde acciones con contenido evaluativo agregado hasta estados mentales o perspectivas de los protagonistas.

A partir de estos resultados puede suponerse que las prácticas pedagógicas de cada enfoque influyeron en el modo de relatar la historia. Es probable que estas diferencias no sólo se circunscriban a las prácticas explícitas, sino también a los modos de interactuar con pares y educadoras que se ven favorecidos en enfoques pedagógicos menos formales (Castillo, 2015), así como las diferentes expectativas que los educadores tienen de los niños, y las que los niños tienen de los educadores y de sí mismos en los diferentes enfoques (Olson y Bruner, 1996). Algunas de estas expectativas como la intención de no producir errores o de evitarlos, no solo se restringen a lo que los educadores proponen, sino que también forman parte de lo que los niños creen que se les pide hacer (Wertsch, 1984).

La propuesta de escribir un texto antes de dominar el sistema de escritura es similar a las prácticas de las participan niños que se educan con un enfoque constructivista, mientras que es poco frecuente cuando el enfoque es formal. Por eso, la misma propuesta pudo haber sido interpretada de diferente modo por quienes suelen escribir textos desde un comienzo, que por quienes solo escriben si están seguros de hacerlo sin errores. Así también el dictado a un 
adulto se interpretaría de un modo diferente cuando esta práctica es familiar que cuando no lo es.

Finalmente, el supuesto teórico que los niños aprenden antes de comenzar la escuela fue fundamental para la constitución de la alfabetización como objeto de investigación. Sin embargo, debido a que los niños también aprenden en las escuelas, es importante considerar el impacto de las prácticas educativas en la alfabetización. El análisis presentado se concentró en aspectos particulares de la producción de textos en contextos educativos diferentes. Es importante continuar ampliando las investigaciones acerca del impacto de las prácticas educativas y los supuestos sobre los que ellas se basan en la alfabetización en sentido amplio. Estas investigaciones son fundamentales para poder crear enlaces y marcos comunes de reflexión entre la investigación psicogenética y la didáctica.

\section{Agradecimientos}

A la Dra. Emilia Ferreiro y a María Claudia Molinari por sus aportes a la investigación. Nuestro especial agradecimiento al Dr. Amihai Rigbi, del Beit Berl College, en Israel por su asesoramiento en el análisis estadístico. Agradecemos también a los dos lectores anónimos que han evaluado una versión previa de este artículo.

\section{Referencias}

Astington, J. W. (2000). Language and metalanguage in children's understanding of mind. En J. W. Astington (ed.), Minds in the making: Essays in honor of David R. Olson. Oxford, UK: Blackwell.

Bamberg, M., y Damrad-Frye, R. (1991). On the ability to provide evaluative comments: Further explorations of children's narrative competencies. Journal of Child Language $18,689-710$.

Berman, R. A., y Slobin, D. I. (eds.). (1994). Relating events in narrative: A crosslinguistic developmental perspective. Hillsdayle, NJ: Lawrence Erlbaum.

Betancourt, Y. M., y Montes, R. G. (2013). Recursos lingüísticos evaluativos en narrativas de experiencia personal: onomatopeyas, interjecciones, repeticiones, verbos y diminutivos. En A. Auza Benavides, y K. Hess Zimmermann, (comp.), ¿Qué me cuentas? Narraciones y desarrollo lingüistico en niños hispano hablantes (pp. 141212). México: DeLaurel. 
Blanche-Benveniste, C. (1998). La escritura del lenguaje dominguero. En E. Ferreiro, y M. Gomez Palacios (comp.), Nuevas perspectivas sobre los procesos de lectura y escritura (pp. 247-270). México: Siglo XXI.

Bosque, I., y Demonte, V. (eds.) (1999). Gramática descriptiva de la lengua española (1r ed., Vol. 3). Madrid: Espasa Calpe.

Brockmeier, J., y Olson, D. R. (2002). Introduction: What is a culture of literacy? En J. Brockmeier, J. M. Wang, y D. R. Olson (eds.), Literacy, narrative and culture (pp. 115). Londres: Curzon.

Bruner, J. (2002). Narrative distancing: A foundation of literacy. En J. Brockmeier, M. Wang, y D. R. Olson (eds.), Literacy, narrative and culture (pp. 86-93). Richmond, Surrey: Curzon Press.

Castedo, M, (2001). Escribir sobre sí mismo como si fuera otro. Revisión de la posición del sujeto de la enunciación en la producción de un texto descriptivo donde el autor es personaje. Rassegna di Psicologia. 18(1), 107-132.

Castillo, C. (2015). Efecto de las prácticas docentes y el nivel de conocimiento letrado de los alumnos al comienzo de la escolaridad en las interacciones de aula. Cultura $y$ Educación, 27(1), 125-157. DOI: http://doi.org/10.1080/11356405.2015.1012805

Cekaite, A., Blum Kulka, S., Grover, V., y Teubal, E. (2014). Introduction. En A. Cekaite, S. Blum Kulka, V. Grover, y E. Teubal (eds.), Children's peer talk (pp. 1-3). Cambridge: Cambridge University Press.

de la Vega Arias, T. J., y Alvarado Castellanos, M. (2013). ¿Te leo un cuento? La lectura de relatos en el desarrollo narrativo de niños en edad preescolar. En A. Auza Benavides, y K. Hess Zimmermann (comp.), ¿Qué me cuentas? Narraciones y desarrollo lingüístico (pp. 173-212). México: DeLaurel.

Ferreiro, E., Pontecorvo, C., Ribeiro Moreira, N., y García Hidalgo, I. (1996). Caperucita roja aprende a escribir. Barcelona: Gedisa.

Ferreiro, E., y Teberosky, A. (1979). Los sistemas de escritura en el desarrollo del niño. México: Siglo XXI.

Grunfeld, D., y Molinari, M. C. (2017). La importancia de pensar sobre la escritura en el jardín de infantes. Magis Revista de Investigación en Educación, 9(19), 31-48.

Heath, S. B. (1983). Ways with words: Language, life and work in communities and classrooms. Cambridge: Cambridge University Press. 
Hess Zimmermann, K., y. Prado, M. L. (2013). ¿Te leo un cuento? La lectura de relatos en el desarrollo narrativo de niños en edad preescolar. En A. Auza Benavides, y K. Hess Zimmermann (comp.), ¿Qué me cuentas? Narraciones y desarrollo lingüístico (pp. 111-140). México: DeLaurel.

Homer. B. D. (2009). Literacy and metalinguistic development. En D. R. Olson y N. Torrance (eds.), The Cambridge handbook of literacy (pp. 487-500). Cambridge: Cambridge University Press.

Hoey, M. (2001). Textual interaction. An introduction to written discourse analysis. Londres: Routledge.

Kuntay, A., y Nakamura, K. (2004). Linguistic Strategies serving evaluative function: a comparison between Japanese and Turkish narratives. En Stromqvist, S, y L. Verthoven (eds.), Relating events in narrative, volume 2: Typological and contextual perspectives (pp. 329-358). Nueva York: Lawrence Erlbaum.

Kupersmitt, J., y Sandbank, A. (2010). Evaluative density in Spanish and Hebrew written fables: a developmental study of forms and function. Paper presented at 6th International Conference on Language Acquisition, Barcelona, Spain.

Labov, W., y Waletsky, J. (1967). Narrative analysis: Oral versions of personal experience. En J. Helms (ed.), Essays on the verbal and visual arts (pp. 12-44). Seattle: University of Washington Press.

Myhill, D., y Jones, D. (2009). How talk becomes text: investigating the concept of oral rehearsal in early years' classrooms. British Journal of Educational Studies, 57(3), 265-284. DOI: http://doi.org/10.1111/j.1467-8527.2009.00438

Nelson, K. (2014). El camino desde la primera infancia a la comunidad de mentes compartidas. Infancia y Aprendizaje, 37, 1-24. DOI: http://doi.org/10.1080/02103702.2014.881654

Nicolopoulou, A. (2008). The elementary forms of narrative coherence in young children's storytelling. Narrative Inquiry, 18(2), 299-325. DOI: http://doi.org/10.1075/ni.18.1.07nic

Olson, D. R., y Bruner, J. S. (1996). Folk psychology and folk pedagogy. En D. R. Olson (ed.), The handbook of education and human development. (pp. 9-27). Malden, Mass.: Blackwell Publishing. 
Pontecorvo, C., \& Orsolini, M. (1996). Writing and written language in children's development. In C. Pontecorvo, M. Orsolini, M. Burge, \& L. Resnik (eds.), Children's early text construction. New Jersey: Lawrence Erlbaum Association.

Pontecorvo, C., y Rossi, F. (2012). The onset of syllabic and alphabetic writings in two groups of five -years old Italian preschool children. En C. Gelatti, B. Arfè, y L. Mason (eds), Issues in writing research (pp. 45-57). Padova: Cooperativa Libraria

Quino (Lavado, J. S.) (2011). Mafalda todas las tiras. España: Lumen

Ravid, D., y Tolchinsky, L. (2002). Developing linguistic literacy: A comprehensive model. Journal of Child Language, 29, 417-447.

Reinhart, T. (1985). From text to meaning: Strategies of evaluation. En Y. Shen (ed.), Cognitive aspects of narrative structure (pp. 4-37). Tel Aviv: Tel Aviv University: The Porter institute.

Richner, E. S., y Nicolopoulou, A. (2001). The narrative construction of differing conceptions of the person in the development of young children's social understanding. Early Education y Development, 12, 393-432.

Rossi, F. Pontecorvo, C., López-Orós, M., y Teberosky, A. (2000). Referential development in storytelling and in storywriting of Catalan and Italian children. Language and Education, 14(3), 164-183. DOI: http://doi.org/10.1080/09500780008666788

Sandbank, A. (2001). On the interplay of genre and writing conventions in early text writing. En L. Tolchinsky, (ed.), Developmental aspects in learning to write (pp. 55-76). Dodrecht: Kluwer.

Sepúlveda, A., y Teberosky, A. (2011). El lenguaje en primer plano en la literatura infantil para la enseñanza y el aprendizaje inicial del lenguaje Escrito. Cultura y Educación, 23 (1), 23-42. DOI: http://doi.org/10.1174/113564011794728560

Shiro, M. (2003). Genre and evaluation in narrative development. Journal of Child Language, 30, 165-195.

Snow, C. E. (2017). The role of vocabulary versus knowledge in children's language learning: a fifty-year perspective / El papel del vocabulario frente al conocimiento en el aprendizaje lingüístico de los niños: una perspectiva de cincuenta años. Infancia y Aprendizaje, 40(1), 1-18. DOI: http://doi.org/10.1080/02103702.2016.1263449

Sulzby, E. (1994). Children's emergent reading of favorite storybooks with postscript. En R. B. Rudell, M. R. Rudell, y H. Singer (eds.), Theoretical models and processes of reading (pp. 244-280). Newark: International Reading Association. 
Tolchinsky, L. (2003). The cradle of culture. Mahwah: Lawrence Erlbaum.

Tolchinsky, L., Bigas, M., y Barragán, C. (2012). Pedagogical practices in the teaching of early literacy in Spain: Voices from the classroom and from the official curricula. Research Papers in Education, 27, 41-62. DOI: http://doi.org/10.1080/02671520903428580

Veneziano, E. (2016). The development on narrative discourse in French by 5 to 10 years old children: Some insights from a conversational interaction method. En J. Perera, M. Aparici, E. Rosado, y N. Salas (eds.), Written and spoken language development across the lifespan (pp. 141-159). Dodrecht: Springer.

Wells, G. (2009). The meaning makers. Learning to talk and talking to learn $2^{\text {nd }}$ edition (pp. 59-73). Bristol, Reino Unido: British library catalogues.

Wertsch, J. V. (1984). The zone of proximal development. In B. Rogoff, \& J.V. Wertsch (eds.), Children's learning in the Zone of Proximal Development. San Francisco: Josey Bass.

Zuccalá, G. (2014). Producir textos en los inicios de la escolaridad: La producción de textos orales, dictados a un adulto y escritos, en sala de 5 y primer grado (Tesis de magíster). Universidad Nacional de La Plata, Argentina.

\footnotetext{
${ }^{1}$ El presente artículo forma parte de la tesis "Producir textos en los inicios de la escolaridad: la producción de textos orales, dictados a un adulto y escritos en sala de 5 y primer grado. Comparación entre enfoques pedagógicos diferentes" de Gabriela Zuccalá, dirigida por la Dra Ana Sandbank en el marco de la Maestría en Escritura y Alfabetización de la Universidad Nacional de La Plata, Argentina.

${ }^{2}$ La Educación Básica en la República Argentina incluye el Nivel Inicial y el Nivel Primario. Los niños entrevistados pertenecen al último año de Nivel Inicial (sala de 5) y el primer año de Nivel primario (Primer grado). Se tomaron estos dos puntos de la escolarización porque allí es donde se comienza con la enseñanza de la lengua escrita y el sistema de escritura. Una presentación detallada de las prácticas se presenta en Zuccalá, 2014.

${ }^{3}$ Una presentación detallada de las prácticas se presenta en Zuccalá, 2014.

${ }^{4} \mathrm{Al}$ presentar las consignas a los niños fueron utilizadas las formas verbales del voceo, propias del habla cotidiana en Argentina.

${ }^{5}$ Los datos presentados a continuación de los ejemplos incluyen: parte del nombre del participante, género: $\mathrm{f}=$ femenino y $\mathrm{m}=$ masculino, nivel $(\mathrm{I}=$ inicial $\mathrm{P}=$-primaria), enfoque: Constructivista o Formal y la modalidad: oral, dictado o escrito.

${ }^{6}$ Una explicación exhaustiva de los niveles de escritura excede el tópico de este artículo, para una caracterización de la escritura ver Ferreiro y Teberosky, 1979; Tolchinsky, 2003.

${ }^{7} \mathrm{Se}$ considera cláusula a una unidad sintáctica que contiene un predicado que expresa una única situación, sea esta una actividad, evento o estado (Berman y Slobin, 1994).
}

\section{Información sobre las autoras:}

Gabriela Zuccalá es Docente de Enseñanza Primaria por la Escuela Normal Nacional Alejandro Carbo y Licenciada en Educación por la Universidad Nacional de Córdoba. En 2015, Gabriela obtuvo el grado de Magíster en Escritura y Alfabetización en la Facultad de Humanidades y Ciencias de la Educación, de la Universidad Nacional de La Plata. Su tesis exploró la expresión evaluativa en narraciones orales, dictadas y escritas, con niños en proceso de alfabetización. Desde 2003, es directora pedagógica de la Escuela Del 
Jacarandá en la ciudad de Buenos Aires. Fue docente de las Cátedras de Epistemología Genética e Historia de la Educación Argentina en la Universidad Nacional de Buenos Aires.

Email: gabrielazuccala@yahoo.com.ar

Dra. Ana Sandbank es docente de la Facultad de Educación en el área de alfabetización inicial en el Levinsky College of Education y en el Beit Berl Academic College, Israel. Su investigación se centra en la escritura de niños en el período de la educación inicial: interacciones entre pares, así como entre niños y educadoras. Ha dictado seminarios acerca de la escritura de textos narrativos en el marco de la Maestría y Especialización en Escritura y Alfabetización en la Universidad Nacional de La Plata Argentina (2009 y 2011).

Email: anasandbank@gmail.com

Para citar este artículo:

Zuccalá, G. y Sandbank, A. (2018). Producir textos en los inicios de la alfabetización: el impacto de diferentes enfoques pedagógicos en la expresión evaluativa. Bellaterra Journal of Teaching \& Learning Language \& Literature, 11(2), 54-73. DOI: http://doi.org/10.5565/rev/jt13.771 